\title{
Biomarkers for Early Detection of Liver Cancer: Focus on Clinical Evaluation
}

\author{
Stella Sun ${ }^{1, *}$, Philip J.R. Day ${ }^{2}$, Nikki P. Lee ${ }^{1}$ and John M. Luk ${ }^{1}$ \\ ${ }^{I}$ Department of Surgery, LKS Faculty of Medicine, Jockey Club Clinical Research Centre, The University of Hong \\ Kong, Pokfulam, Hong Kong, ${ }^{2}$ Interdisciplinary Molecular Medicine, The Manchester Interdisciplinary Biocentre, Uni- \\ versity of Manchester, Manchester, UK
}

\begin{abstract}
This review summarises the screening methods from hepatic ultrasonography to serological biomarkers for early detection of liver cancer and focuses on evaluation of biomarkers ability. The development of novel biomarkers according to the 5-phase program defined by the Early Detection Research Network (EDRN) is also outlined in this review.
\end{abstract}

Keywords: Hepatocellular carcinoma, early cancer detection network, diagnostic imaging, serological biomarkers.

\section{INTRODUCTION}

Metastasis always presents a major challenge before neoplasm is finally conquered and once the malignant state is acquired, the morbidity and mortality commonly becomes high and less likely to benefit from therapeutic intervention [1]. The prognosis of different neoplasm, e.g., HCC [2], lung [3], ovarian [4] and prostate cancer [5] is poor and has hardly improved in recent years, largely owing to the lack of early diagnosis, frequent recurrence after surgery and poor surveillance programs.

Early detection of cancer ideally helps to decrease the mortality rate, resulting in more effective treatment and prolonged survival [6]. However, in HCC, prognosis remains dismal because of its tumour aggressiveness and the frequent association with the common risk factor cirrhosis, which renders curative treatment impossible even if it is detected at an early stage. The recent practice in HCC and in some neoplastic states commonly employed screening regimens for early detection. This tool has already been applied in routine surveillance for $\mathrm{HCC}$ in high risk patients with chronic viral hepatitis or cirrhosis. The benefits of early diagnosis have prompted research into different methods of screening, which include the form of serological biomarkers or radiological tests [7]. Previous studies suggested HCC patients acquired better survival when detected by screening for $\mathrm{HCC}$ in high-risk patients [8]. This review summarises the current available screening tools for HCC surveillance both radiologically and serologically, and to evaluate the sensitivity and specificity of different biomarkers as the screening regimens for early detection of HCC. Finally, it discusses the proposed 5-phase programs by the National Cancer Institute in order to framework the structure of new biomarkers' development.

\section{SCREENING BY RADIOLOGICAL IMAGING}

In carcinogenesis of cirrhotic liver to the development of early $\mathrm{HCC}$, different phases from benign regenerative nodule

\footnotetext{
*Address correspondence to this author at the Department of Surgery, The University of Hong Kong, 21 Sassoon Road, Pokfulam, Hong Kong; Fax: (852) 28199636; E-mail: sunstella1980@yahoo.co.uk
}

through low-grade dysplastic nodules (LGDN) to high-grade dysplastic nodules (HGDN) are commonly observed before the final development into overt HCC. Recent advances in diagnostic imaging allows early detection of HCC by screening of high-risk populations [9] and the diagnostic outcome is heavily dependent on imaging characteristics. For instance, small HCCs are mostly nodular lesions, large HCCs are classified with expansive nodular growth patterns, infiltrative or diffuse and well-differentiated HCCs often show a fibrous capsule. However, limitation on imaging modalities presents a problem in the currently used primary radiologic screening platforms [10] for small HCC diagnosis.

The American Association for the Study of Liver Disease (AASLD) has recently recommended that diagnosis of HCC can be made if a tumour size larger than $2 \mathrm{~cm}$ shows typical features of HCC in material-enhanced computed tomography (CT) or magnetic resonance (MR) imaging [11]. However, there is always difficulty in imaging the differentiation of precancerous lesions and characterization of hypervascular nodules smaller than $2 \mathrm{~cm}$. The context below is a brief evaluation of the effectiveness of different imaging modalities (Table 1).

Ultrasound (US), computed tomography (CT), as well as modern magnetic resonance imaging are the commonly used non-invasive, diagnostic imaging techniques for HCC. However, each of the techniques is limited by a heterogeneous sensitivity and specificity. For instance, ultrasound method has been shown to have low sensitivity but high specificity in revealing $\mathrm{HCC}$ in patients who already have a cirrhotic liver [12]. Bennett GL's group demonstrated that the overall sensitivity of small lesion tumours is only $20.5 \%$, to a maximum of $75 \%$ for larger lesions of more than $5 \mathrm{~cm}$ in size [13]. Limitation of the conventional ultrasound could be improved by contrast-enhanced (CE) ultrasonography by using ultrasound contrast agents such as perfluorocarbon or sulphur hexafluoride.

CT and MRI are the other two commonly used diagnostic imaging techniques in $\mathrm{HCC}$; their predictive values for screening patients with cirrhosis and no known HCC are relatively low at around $50 \%$ detection sensitivity. In es- 
Table 1. Diagnostic Performance of Different Radiological Platforms

\begin{tabular}{|c|c|c|}
\hline Radiological Imaging & Sensitivity & Reference \\
\hline \hline CT & Overall sensitivity for early HCC detection is 56\% & Takayasu, K et al 2007 [46] \\
\hline MDCT & Detection rate for small HCC tumours is $97.5 \%$ & Zhao, H et al 2007 [47] \\
\hline CTA & Detection sensitivity of $>80 \%$ in HCC nodules smaller than 2cm & Kim, SR et al 2007 [48] \\
\hline US & $\begin{array}{c}\text { Overall detection sensitivity for HCC is } 20.5 \% \text { and a maximum of } 75 \% \text { for larger } \\
\text { lesions of more than 5cm in size. }\end{array}$ & Benett, GL et al 2002 [13] \\
\hline CEUS & $\begin{array}{c}\text { Sensitivity in the detection of arterial hyperenhancement is } 91 \% \text {. Diagnostic sensi- } \\
\text { tivity of HCC in 2cm nodules or smaller is 52\%-91\%. }\end{array}$ & Gaiani, S et al 2004 [49] \\
\hline MRI & $\begin{array}{c}\text { Sensitivity of } 90 \% \text { in the presence of arterial enhancement. Tumours larger than } \\
2 \mathrm{~cm} \text { of detection sensitivity }>90 \% \text { whereas in tumours smaller than 2cm is 33\% }\end{array}$ & El-Serag, HB et al 2008 [50] \\
\hline PDG-PET & $\begin{array}{c}\text { Detection sensitivity of well-differentiated HCC is low, and sensitivity in diagnos- } \\
\text { ing HCC is } 55 \%\end{array}$ & Khan, MA et al 2000 [51] \\
\hline
\end{tabular}

Abbreviation used: CT, computed tomography; MDCT, multidetector computed tomography; CTA, CT arteriography; US, ultrasound; CEUS, contrast-enhanced ultrasound; MRI, magnetic resonance imaging; PDG-PET, positron-emission-tomography-computed tomography.

sence, the diagnosis is often missed in tumours smaller than $2 \mathrm{~cm}$ and those well differentiated tumours [14]. In addition, the sensitivity of both CT and MRI are poorly defined for the detection of dysplastic nodules in cirrhotic background patients.

Although other contrast enhanced CT and MRI have been introduced to characterize the definitive distinction between early stage, well-differentiated HCC and borderline lesions, there is still a lack of competence in diagnosis of early HCC in nodules smaller than $2 \mathrm{~cm}$. Diagnosis of HCC may be positively established non-invasively by a combination of history, physical assessment, blood tests and radiology. Therefore, postulation of an alternative screening strategy by serological markers is supplement to the routine surveillance for HCC.

\section{SCREENING BY SEROLOGICAL BIOMARKERS}

New biomarkers are being discovered to identify individuals at risk of cancer, i.e. for early diagnosis, to determine prognosis, to detect recurrence and to monitor treatment [15]. Marrero JA et al described that the ideal biomarkers should be sensitive, specific, non-invasive, reproducible and most importantly enable detection of HCC at an early stage [7]. Although early detection is a key step for effective intervention of $\mathrm{HCC}$, the lack of sensitive and specific biomarkers is a major reason for the high rate of HCC-related mortality [16]. Currently, surveillance involves both serological testing with serum AFP estimation and ultrasound scanning, typically at 6-monthly intervals [17]. Serum biomarkers are considered to be the effective method for early detection of HCC and compensate for the symptomatic problems associated with radiological diagnosis. Serological biomarkers could be divided into 4 categories: (i) oncofetal antigens and glycoprotein antigens; (ii) enzymes and isoenzymes; (iii) genes; growth factor and (iv) cytokines [18]. The following categories discuss the most widely applied tumour markers for single or combinational use in order to improve the effectiveness for screening HCC patients (Table 2).

\section{ONCOFETAL AND GLYCOPROTEIN ANTIGENS}

AFP is the most commonly used serum marker for routine surveillance of HCC, however there are limitations of AFP when applied in patients with different etiopathological origins. Poor sensitivity and specificity of AFP has been reported in different studies [19] and elevated serum AFP levels are noted in patients with chronic hepatitis $\mathrm{C}$ without HCC [20]. The reported sensitivity and specificity of AFP are $39 \%$ to $65 \%$ and $65 \%$ to $94 \%$, respectively [10]. Moreover, while AFP may be a useful diagnostic serum marker in patients with advanced neoplasm, it has limited utility as a screening surveillance in patients with early or small tumours with reduced detection sensitivity [21]. Although the conventional marker AFP has limited sensitivity and specificity, the serum isoforms of AFP based on differential lectin binding of the glycan moiety appear to be more effective than AFP in differentiating HCC from non-malignant hepatopathy and for detecting small HCC tumours. For instance, lens culinaris agglutinin - reactive AFP (AFP-L3) is the major glycoform of AFP recently reported to be related to the progression from moderately differentiated to poorly differentiated HCC [22]. AFP-L3 levels had sensitivities and specificities that ranged from $36 \%$ to $96 \%$ and $89 \%$ to $94 \%$, respectively. Researchers now attempt to correlate the efficacy of different markers as single or combinational use with respect to size and tumour burden. AFP-L3 is commonly correlated or compared with levels of AFP and des-gammacarboxyprothrombin (DCP). However, a recent report suggested that no improved efficacy was observed by combining the two or three markers for HCC diagnosis [23].

Golgi protein 73 (GP 73) is another biomarker belonging to the glycoprotein antigen group and has recently been found to be elevated significantly in HCC patients compared to those with cirrhosis. GP73 is an integral membrane protein localized to the cis Golgi and functions in assisting proteins synthesis and transportation. Proprotein convertase mediated cleavage resulting in GP73 secretion [24]. In human liver tissues, GP73 was expressed in biliary epithelial 
Table 2. Comparison of the Performance of Single and Combined Diagnostic Markers in HCC

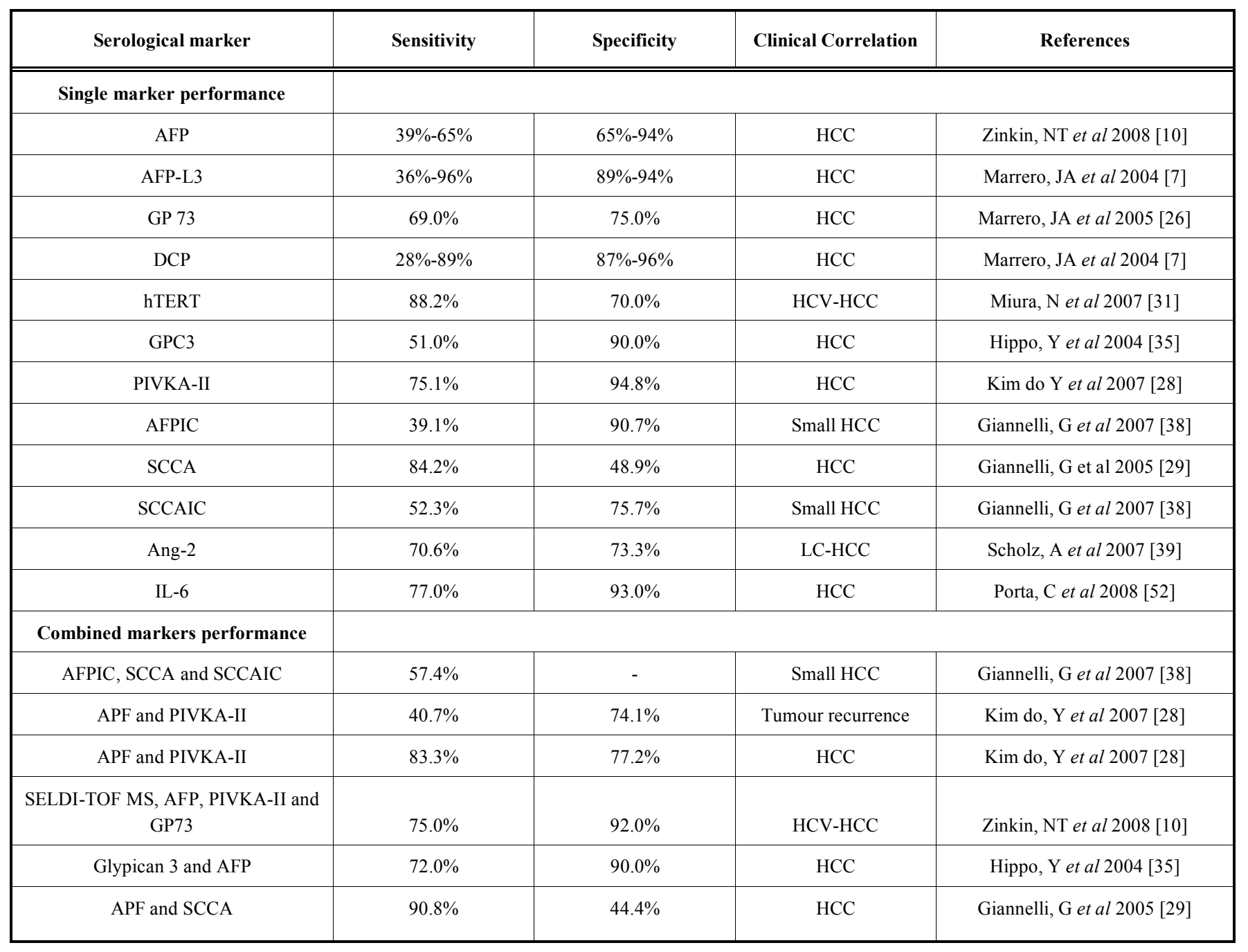

cells, normal hepatocytes exhibited weak expression signal whereas strong expression of GP73 was found in hepatitis liver [25]. Exploration by targeted glycoproteomics of human serum glycoprotein proteome showed GP73 was elevated and hyperfucosylated in animals with HCC. Furthermore, GP73 has demonstrated to be up-regulated in the serum of $\mathrm{HCC}$ patients with $\mathrm{HBV}$ infected origin, but not in $\mathrm{HBV}$ or HCV infected individuals. Immunoblots and densitometric quantitation of serum in $353 \mathrm{HCC}$ patients confirmed that serum GP73 levels were significantly higher in patients with HCC compared to those with cirrhosis, with suggested sensitivity of $69 \%$ and specificity of $75 \%$. More importantly, GP73 levels showed a better sensitivity (62\%) than AFP (25\%) for early detection of HCC [26].

\section{ENZYMES AND ISOENZYMES}

Des- $\gamma$ carboxyprothrombin, also known as prothrombin is induced by vitamin $\mathrm{K}$ absence-II (PIVKA-II), which has been reported as a better biomarker than AFP in differentiating HCC from non-neoplastic state and allows small HCC tumour detection [18]. DCP has sensitivities and specificities that ranged from $28 \%$ to $89 \%$ and $87 \%$ to $96 \%$, respectively
[7]. The prevalence of elevated levels of these tumour markers increase with progression of tumour stage in HCC patients, also survival was poorer among patients with elevated levels of these markers [27]. Recently, PIVKA-II has been reported as a useful tumour marker for HCC; complementary to AFP, PIVKA-II might be helpful for early diagnosis of tumour recurrence [28] and may potentially act as a useful indicator of vascular invasion [22]. A report on performance characteristics evaluation of AFP, AFP-L3 and DCP suggested that DCP is significantly better than total AFP or AFP-L3 in differentiating HCC from cirrhosis. Different studies are now under investigation to evaluate the clinical usefulness of these biomarkers. There are also studies performed to evaluate the combine use of different markers in order to increase the sensitivity and specificity for early HCC detection $[29,30]$.

\section{GENES}

Human telomerase reverse transcriptase (hTERT) mRNA is a recent candidate, other than a protein, proved to be superior to AFP and DCP in HCC diagnosis. hTERT mRNA has a higher expression in HCC patients' serum than those with chronic liver diseases. hTERT mRNA expression independ- 
ently correlated with tumour differentiation. It has been reported that the sensitivity and specificity of hTERT mRNA in detecting $\mathrm{HCC}$ is $88.2 \%$ and $70 \%$, respectively. More importantly, hTERT surpasses AFP and DCP in the diagnosis of HCC [31]. AFP mRNA in blood reflects the presence of circulating HCC cells and indicates the presence of metastasis. However, AFP mRNA is frequently detected in the blood despite only benign liver diseases being present and even when patients have no clinical evidence of extrahepatic metastases. Therefore, the specificity of the AFP mRNA as HCC biomarker and its correlation with the clinicalpathological parameters of HCC remains controversial [32].

\section{GROWTH FACTOR AND CYTOKINES}

The two well-documented biomarkers in this group include brain-derived neurotrophic factor (BDNF) and glypican-3 (GPC3). Glypican-3, a heparan sulfate proteoglycan, plays an important role in cell growth, differentiation and migration. Overexpression of GPC 3 mRNA and protein has been reported in both serum and tissues of early-stage HCC but not in normal hepatic tissues [33]. Immunocytochemical staining for GPC3 distinguishes HCC from benign and malignant hepatic lesions and the reported sensitivity and specificity of glypican-3 in HCC was $90 \%$ and $100 \%$ respectively [34]. In addition, glypican-3 was undetectable in the serum of healthy donors and patients with non-malignant liver disease, whereas in HCC patients glypican-3 was increased to $53 \%$ of HCC patients [33]. Furthermore, GPC3 showed superior sensitivity over AFP in well- or moderatelydifferentiated HCC and the combination of these two markers improved overall sensitivity from $50 \%$ to $72 \%$ [35]. So far, results revealed that glypican-3 is a promising serological marker for early detection of HCC. BDNF is a member of the nerve growth factor family, over-expression of this protein was found to be related to HCC development and recurrence [36]. The serum levels of BDNF were positively correlated with the platelet counts in HCC patients, suggesting that the interaction between serum BDNF and platelets might play an important role in HCC tumour progression [37]. However, there has been no report on the sensitivity and specificity of this marker for early detection of HCC.

Recently, many research groups have reported the different combined use of serum biomarkers in clinical practice in order to increase the accuracy of HCC diagnosis. For instance, Squamous Cell Carcinoma Antigen (SCCA), a serine protease inhibitor physiologically present in the skin, and also the immunocomplexed (IC) forms of SCCA and AFP: SCCAIC and AFPIC, respectively [38]. In addition, the use of angiopoietin-2 in combination with AFP level led to improve discrimination between $\mathrm{HCC}$ and cirrhosis. Angiopoietin-2 (Ang-2) serum levels were found to be elevated in patients with liver cirrhosis and $\mathrm{HCC}$, implicating a possible role of the angiopoietin-Tie-2 system for neoangiogenesis in cirrhosis as well as a marker for the detection of cirrhosis and HCC [39].

In essence, the positive rate of currently available tumour markers can not surpass abdominal ultrasonography (US) as modalities to detect small HCC at an early stage, which results in a possible delay of its diagnosis. Moreover, there is always a need to develop more sensitive markers for early detection of HCC. Herein, we outline the guidelines for the process of new biomarker evaluation and development in a 5 phase program.

\section{BIOMARKERS EVALUATION AND DEVELOPMENT}

The Early Detection Research Network (EDRN) of the National Cancer Institute has established a formal framework to guide the process of biomarker evaluation and development in a 5-phase program [40]. The purpose of the EDRN is to framework a guide for biomarker development by coordinating ongoing research on biomarker- screening, biomarkervalidation, clinical repositories and population-screening programs in order to promote efficiency and to enhance early detection of cancer. Moreover, the screening tool must be sufficiently non-invasive and inexpensive to allow widespread applicability. An ideal biomarker should be able to detect specifically and non-invasively in tumours but not in other non-neoplastic states. As many cancers are heterogeneous, especially $\mathrm{HCC}$, it is rare that a single biomarker could be used for detection in high specificity and sensitivity. Therefore, the latest research trend is to apply a panel of biomarkers, so as to increase both detection sensitivity and specificity.

Recent development in microarrays, proteomics and immunology offers new approaches to cancer screening [4143]. Phase 1- Preclinical Exploratory Studies proposed biomarker selection in preclinical studies by identifying characteristics unique to tumour tissues that might give ideas in clinical tests for detecting cancer. Thereafter, phase II is entered by performing clinical assay and validation in order to identify biomarkers that can distinguish subjects with cancer from those without cancer. An important aspect of phase 2 studies is to identify biomarkers that can detect early-stage cancer, therefore, the specimen used for biomarker assay must be obtained non-invasively. Inclusion of an adequate number of cases in the early stage is important to determine the diagnostic capability of the biomarker for early-stage cancer [7]. Evaluation on combined use of panels of biomarkers is always important in this phase. Many currently employed biomarkers have been identified in this stage, for instance; lens culinaris agglutintin - reactive AFP (AFP-L3) and des-gamma carboxyprothrombin (DCP). These have been studied extensively and appear to be effective in HCC.

Prior to the prospective screening, phase 3-Retrospective Longitudinal Repository Studies is carried out as to evaluate the capacity of biomarkers to detect preclinical disease. This involves the collection of specimens with no clinical diagnosis and to determine whether the biomarker can detect cancer cases in order to test the capability of pre-clinical disease detection. ROC statistical analysis is commonly used in this phase to evaluate the performance of a biomarker to identify high-risk subjects destined to develop cancer. Markers identified in this phase include insulin-like growth factor-1 (IGF1) [44] and human HGF [45]; however, evaluation of these markers has been limited in a few studies. Further evaluation will require a long duration of follow-up in a large cohort of high-risk subjects for more accurate analysis. The retrospective phase 3 study determines whether tumours can be detected early before clinical diagnosis. However, no information can be identified on the stage and nature of the cancer 
by the time it is detected. Therefore, phase 4- Prospective Screening Studies is established to determine the operating characteristics of the biomarkers. Studies at this stage involve individual screening followed by diagnosis and treatment. The final phase addresses whether screening surveillance of high-risk population allows early detection of cancer. The formulated five-phase structure ideally enhances the generation of more promising and effective biomarkers. However, some biomarkers for HCC have still remained in phase 1 or 2 studies although they have been reported as potential markers for over 15 years. Further progression should be performed in biomarker validation and federally funded collaborative research networks should be established so that promising biomarkers identified in phase 1 and 2 studies can be evaluated further [7].

\section{CONCLUDING REMARKS AND FUTURE PERSPEC- TIVES}

The existing biomarkers as well as the newly identified biomarkers have many limitations regarding their sensitivity and specificity. The combinational use of different biomarkers may enhance the detection sensitivity for early detection of HCC. However, further evaluation and development are necessary to enhance the capability of these biomarkers. In order to more effectively assess the development of biomarkers for early detection, the 5 phases program defined by the EDRN may help to evaluate and organise biomarker development for future validation studies.

\section{LIST OF ABBREVIATIONS}

$$
\begin{array}{ll}
\mathrm{HCC} & =\text { Hepatocellular carcinoma } \\
\mathrm{CT} & =\text { Computed tomography } \\
\mathrm{MRI} & =\text { Magnetic resonance imaging } \\
\mathrm{AFP} & =\text { Alpha fetoprotein } \\
\text { AFP-L3 } & \text { Lens culinaris agglutintin - reactive AFP } \\
\mathrm{DCP} & =\text { Des-gamma carboxyprothrombin } \\
\mathrm{IL}-6 & =\text { Interleukin } 6
\end{array}
$$

\section{REFERENCES}

[1] Tang, Z. Y.; Ye, S. L.; Liu, Y. K.; Qin, L. X.; Sun, H. C.; Ye, Q. H.; Wang, L.; Zhou, J.; Qiu, S. J.; Li, Y.; Ji, X. N.; Liu, H.; Xia, J. L.; Wu, Z. Q.; Fan, J.; Ma, Z. C.; Zhou, X. D.; Lin, Z. Y.; Liu, K. D. A decade's studies on metastasis of hepatocellular carcinoma. $J$. Cancer Res. Clin. Oncol., 2004, 130(4), 187-96.

[2] Feng, J. T.; Shang, S.; Beretta, L. Proteomics for the early detection and treatment of hepatocellular carcinoma. Oncogene, 2006, 25(27), 3810-7.

[3] Conrad, D. H.; Goyette, J.; Thomas, P. S. Proteomics as a method for early detection of cancer: a review of proteomics, exhaled breath condensate, and lung cancer screening. J. Gen. Intern. Med., 2008, 23 Suppl 1,78-84.

[4] Visintin, I.; Feng, Z.; Longton, G.; Ward, D. C.; Alvero, A. B.; Lai, Y.; Tenthorey, J.; Leiser, A.; Flores-Saaib, R.; Yu, H.; Azori, M.; Rutherford, T.; Schwartz, P. E.; Mor, G. Diagnostic markers for early detection of ovarian cancer. Clin. Cancer Res., 2008, 14(4), 1065-72.

[5] Laxman, B.; Morris, D. S.; Yu, J.; Siddiqui, J.; Cao, J.; Mehra, R.; Lonigro, R. J.; Tsodikov, A.; Wei, J. T.; Tomlins, S. A.; Chinnaiyan, A. M. A first-generation multiplex biomarker analysis of urine for the early detection of prostate cancer. Cancer Res., 2008, 68(3), 645-9.
[6] Wu, F. S.; Zhao, W. H.; Liang, T. B.; Ma, Z. M.; Teng, L. S.; Wang, M.; Zheng, S. S. Survival factors after resection of small hepatocellular carcinoma. Hepatobiliary Pancreat. Dis. Int., 2005, $4(3), 379-84$.

[7] Marrero, J. A.; Lok, A. S. Newer markers for hepatocellular carcinoma. Gastroenterology, 2004, 127, 5 Suppl 1, S113-9.

[8] Zoli, M.; Magalotti, D.; Bianchi, G.; Gueli, C.; Marchesini, G.; Pisi, E. Efficacy of a surveillance program for early detection of hepatocellular carcinoma. Cancer, 1996, 78(5), 977-85.

[9] Takayama, T.; Makuuchi, M.; Hirohashi, S.; Sakamoto, M.; Yamamoto, J.; Shimada, K.; Kosuge, T.; Okada, S.; Takayasu, K.; Yamasaki, S. Early hepatocellular carcinoma as an entity with a high rate of surgical cure. Hepatology, 1998, 28(5), 1241-6.

[10] Zinkin, N. T.; Grall, F.; Bhaskar, K.; Otu, H. H.; Spentzos, D.; Kalmowitz, B.; Wells, M.; Guerrero, M.; Asara, J. M.; Libermann, T. A.; Afdhal, N. H. Serum Proteomics and Biomarkers in Hepatocellular Carcinoma and Chronic Liver Disease. Clin. Cancer Res., 2008, 14(2), 470-477.

[11] Willatt, J. M.; Hussain, H. K.; Adusumilli, S.; Marrero, J. A. MR Imaging of hepatocellular carcinoma in the cirrhotic liver: challenges and controversies. Radiology, 2008, 247(2), 311-30.

[12] Saar, B.; Kellner-Weldon, F. Radiological diagnosis of hepatocellular carcinoma. Liver Int., 2008, 28(2), 189-99.

[13] Bennett, G. L.; Krinsky, G. A.; Abitbol, R. J.; Kim, S. Y.; Theise, N. D.; Teperman, L. W. Sonographic detection of hepatocellular carcinoma and dysplastic nodules in cirrhosis: correlation of pretransplantation sonography and liver explant pathology in 200 patients. AJR Am. J. Roentgenol., 2002, 179(1), 75-80.

[14] Takayama, T.; Makuuchi, M.; Kojiro, M.; Lauwers, G. Y.; Adams, R. B.; Wilson, S. R.; Jang, H. J.; Charnsangavej, C.; Taouli, B. Early hepatocellular carcinoma: pathology, imaging, and therapy. Ann. Surg. Oncol., 2008, 15(4), 972-8.

[15] Bast, R. C., Jr.; Lilja, H.; Urban, N.; Rimm, D. L.; Fritsche, H.; Gray, J.; Veltri, R.; Klee, G.; Allen, A.; Kim, N.; Gutman, S.; Rubin, M. A.; Hruszkewycz, A. Translational crossroads for biomarkers. Clin. Cancer Res., 2005, $11(17), 6103-8$.

[16] He, Q. Y.; Zhu, R.; Lei, T.; Ng, M. Y.; Luk, J. M.; Sham, P.; Lau, G. K.; Chiu, J. F. Toward the proteomic identification of biomarkers for the prediction of HBV related hepatocellular carcinoma. $J$. Cell Biochem., 2008, 103(3), 740-52.

[17] Trevisani, F.; De, N. S.; Rapaccini, G.; Farinati, F.; Benvegnu, L.; Zoli, M.; Grazi, G. L.; Del, P. P.; Di, N.; Bernardi, M. Semiannual and annual surveillance of cirrhotic patients for hepatocellular carcinoma: effects on cancer stage and patient survival (Italian experience). Am. J. Gastroenterol., 2002, 97(3), 734-44.

[18] Zhou, L.; Liu, J.; Luo, F. Serum tumor markers for detection of hepatocellular carcinoma. World J. Gastroenterol., 2006, 12(8), 1175-81.

[19] Bruix, J.; Sherman, M.; Llovet, J. M.; Beaugrand, M.; Lencioni, R.; Burroughs, A. K.; Christensen, E.; Pagliaro, L.; Colombo, M.; Rodes, J. Clinical management of hepatocellular carcinoma. Conclusions of the Barcelona-2000 EASL conference. European Association for the Study of the Liver. J. Hepatol., 2001, 35(3), 421-30.

[20] Chen, T. M.; Huang, P. T.; Tsai, M. H.; Lin, L. F.; Liu, C. C.; Ho, K. S.; Siauw, C. P.; Chao, P. L.; Tung, J. N. Predictors of alphafetoprotein elevation in patients with chronic hepatitis $\mathrm{C}$, but not hepatocellular carcinoma, and its normalization after pegylated interferon alfa 2a-ribavirin combination therapy. J. Gastroenterol. Hepatol., 2007, 22(5), 669-75.

[21] Mazzaferro, V.; Regalia, E.; Doci, R.; Andreola, S.; Pulvirenti, A.; Bozzetti, F.; Montalto, F.; Ammatuna, M.; Morabito, A.; Gennari, L. Liver transplantation for the treatment of small hepatocellular carcinomas in patients with cirrhosis. N. Engl. J. Med., 1996, 334(11), 693-9.

[22] Miyaaki, H.; Nakashima, O.; Kurogi, M.; Eguchi, K.; Kojiro, M. Lens culinaris agglutinin-reactive alpha-fetoprotein and protein induced by vitamin $\mathrm{K}$ absence II are potential indicators of a poor prognosis: a histopathological study of surgically resected hepatocellular carcinoma. J. Gastroenterol., 2007, 42(12), 962-8.

[23] Durazo, F. A.; Blatt, L. M.; Corey, W. G.; Lin, J. H.; Han, S.; Saab, S.; Busuttil, R. W.; Tong, M. J. Des-gamma-carboxyprothrombin, alpha-fetoprotein and AFP-L3 in patients with chronic hepatitis, cirrhosis and hepatocellular carcinoma. J. Gastroenterol. Hepatol., 2008, 23, 1541-48. 
[24] Bachert, C.; Fimmel, C.; Linstedt, A. D. Endosomal trafficking and proprotein convertase cleavage of cis Golgi protein GP73 produces marker for hepatocellular carcinoma. Traffic, 2007, 8(10), 1415-23.

[25] Kladney, R. D.; Bulla, G. A.; Guo, L.; Mason, A. L.; Tollefson, A. E.; Simon, D. J.; Koutoubi, Z.; Fimmel, C. J. GP73, a novel Golgilocalized protein upregulated by viral infection. Gene, 2000, 249(12), 53-65.

[26] Marrero, J. A.; Romano, P. R.; Nikolaeva, O.; Steel, L.; Mehta, A.; Fimmel, C. J.; Comunale, M. A.; D'Amelio, A.; Lok, A. S.; Block, T. M. GP73, a resident Golgi glycoprotein, is a novel serum marker for hepatocellular carcinoma. J. Hepatol., 2005, 43(6), 1007-12.

[27] Toyoda, H.; Kumada, T.; Osaki, Y.; Oka, H.; Kudo, M. Role of tumor markers in assessment of tumor progression and prediction of outcomes in patients with hepatocellular carcinoma. Hepatol. Res., 2007, 37 Suppl 2, S166-71.

[28] Kim do, Y.; Paik, Y. H.; Ahn, S. H.; Youn, Y. J.; Choi, J. W.; Kim, J. K.; Lee, K. S.; Chon, C. Y.; Han, K. H. PIVKA-II is a useful tumor marker for recurrent hepatocellular carcinoma after surgical resection. Oncology, 2007, 72 Suppl 1, 52-7.

[29] Giannelli, G.; Marinosci, F.; Trerotoli, P.; Volpe, A.; Quaranta, M.; Dentico, P.; Antonaci, S. SCCA antigen combined with alphafetoprotein as serologic markers of HCC. Int. J. Cancer, 2005, 117(3), 506-9.

[30] Izuno, K.; Fujiyama, S.; Yamasaki, K.; Sato, M.; Sato, T. Early detection of hepatocellular carcinoma associated with cirrhosis by combined assay of des-gamma-carboxy prothrombin and alphafetoprotein: a prospective study. Hepatogastroenterology, 1995, 42(4), 387-93.

[31] Miura, N.; Maruyama, S.; Oyama, K.; Horie, Y.; Kohno, M.; Noma, E.; Sakaguchi, S.; Nagashima, M.; Kudo, M.; Kishimoto, Y.; Kawasaki, H.; Hasegawa, J.; Shiota, G. Development of a novel assay to quantify serum human telomerase reverse transcriptase messenger RNA and its significance as a tumor marker for hepatocellular carcinoma. Oncology, 2007, 72 Suppl 1, 45-51.

[32] Cillo, U.; Vitale, A.; Navaglia, F.; Basso, D.; Montin, U.; Bassanello, M.; D'Amico, F.; Ciarleglio, F. A.; Brolese, A.; Zanus, G.; De Pascale, V.; Plebani, M.; D'Amico, D. F. Role of blood AFP mRNA and tumor grade in the preoperative prognostic evaluation of patients with hepatocellular carcinoma. World J. Gastroenterol., 2005, 11(44), 6920-5.

[33] Capurro, M.; Wanless, I. R.; Sherman, M.; Deboer, G.; Shi, W.; Miyoshi, E.; Filmus, J. Glypican-3: a novel serum and histochemical marker for hepatocellular carcinoma. Gastroenterology, 2003, 125(1), 89-97.

[34] Kandil, D.; Leiman, G.; Allegretta, M.; Trotman, W.; Pantanowitz, L.; Goulart, R.; Evans, M. Glypican-3 immunocytochemistry in liver fine-needle aspirates : a novel stain to assist in the differentiation of benign and malignant liver lesions. Cancer, 2007, 111(5), 316-22.

[35] Hippo, Y.; Watanabe, K.; Watanabe, A.; Midorikawa, Y.; Yamamoto, S.; Ihara, S.; Tokita, S.; Iwanari, H.; Ito, Y.; Nakano, K.; Nezu, J.; Tsunoda, H.; Yoshino, T.; Ohizumi, I.; Tsuchiya, M.; Ohnishi, S.; Makuuchi, M.; Hamakubo, T.; Kodama, T.; Aburatani, H. Identification of soluble NH2-terminal fragment of glypican-3 as a serological marker for early-stage hepatocellular carcinoma. Cancer Res., 2004, 64(7), 2418-23.

[36] Yang, Z. F.; Ho, D. W.; Lam, C. T.; Luk, J. M.; Lum, C. T.; Yu, W. C.; Poon, R. T.; Fan, S. T. Identification of brain-derived neurotrophic factor as a novel functional protein in hepatocellular carcinoma. Cancer Res., 2005, 65(1), 219-25.

[37] Yang, Z. F.; Ho, D. W.; Lau, C. K.; Tam, K. H.; Lam, C. T.; Yu, W. C.; Poon, R. T.; Fan, S. T. Significance of the serum brainderived neurotrophic factor and platelets in hepatocellular carcinoma. Oncol. Rep., 2006, 16(6), 1237-43.

[38] Giannelli, G.; Fransvea, E.; Trerotoli, P.; Beaugrand, M.; Marinosci, F.; Lupo, L.; Nkontchou, G.; Dentico, P.; Antonaci, S. Clinical validation of combined serological biomarkers for improved hepatocellular carcinoma diagnosis in 961 patients. Clin. Chim. Acta., 2007, 383(1-2), 147-52.

[39] Scholz, A.; Rehm, V. A.; Rieke, S.; Derkow, K.; Schulz, P.; Neumann, K.; Koch, I.; Pascu, M.; Wiedenmann, B.; Berg, T.; Schott, E. Angiopoietin-2 serum levels are elevated in patients with liver cirrhosis and hepatocellular carcinoma. Am. J. Gastroenterol., 2007, 102(11), 2471-81.

[40] Pepe, M. S.; Etzioni, R.; Feng, Z.; Potter, J. D.; Thompson, M. L; Thornquist, M.; Winget, M.; Yasui, Y. Phases of biomarker development for early detection of cancer. J Natl. Cancer Inst., 2001, 93(14), 1054-61.

[41] Sun, S.; Lee, N. P.; Poon, R. T.; Fan, S. T.; He, Q. Y.; Lau, G. K.; Luk, J. M. Oncoproteomics of hepatocellular carcinoma: from cancer markers' discovery to functional pathways. Liver Int., 2007, 27(8), 1021-38.

[42] Lee, N. P., Cheung, S. T., Poon, R. T., Fan, S. T. and Luk, J. M. Genomic and proteomic biomarkers for diagnosis and prognosis of hepatocellular carcinoma. Biomarkers Med., 2007, 1, 273-284.

[43] Gagnon, A.; Kim, J. H.; Schorge, J. O.; Ye, B.; Liu, B.; Hasselblatt, K.; Welch, W. R.; Bandera, C. A.; Mok, S. C. Use of a Combination of Approaches to Identify and Validate Relevant TumorAssociated Antigens and Their Corresponding Autoantibodies in Ovarian Cancer Patients. Clin. Cancer Res., 2008, 14(3), 764-771.

[44] Mazziotti, G.; Sorvillo, F.; Morisco, F.; Carbone, A.; Rotondi, M.; Stornaiuolo, G.; Precone, D. F.; Cioffi, M.; Gaeta, G. B.; Caporaso, N.; Carella, C. Serum insulin-like growth factor I evaluation as a useful tool for predicting the risk of developing hepatocellular carcinoma in patients with hepatitis $\mathrm{C}$ virus-related cirrhosis: a prospective study. Cancer, 2002, 95(12), 2539-45.

[45] Yamagamim, H.; Moriyama, M.; Matsumura, H.; Aoki, H.; Shimizu, T.; Saito, T.; Kaneko, M.; Shioda, A.; Tanaka, N.; Arakawa, Y. Serum concentrations of human hepatocyte growth factor is a useful indicator for predicting the occurrence of hepatocellular carcinomas in C-viral chronic liver diseases. Cancer, 2002, 95(4), 82434.

[46] Takayasu, K.; Muramatsu, Y.; Mizuguchi, Y.; Ojima, H. CT Imaging of early hepatocellular carcinoma and the natural outcome of hypoattenuating nodular lesions in chronic liver disease. Oncology, 2007, 72 Suppl 1, 83-91.

[47] Zhao, H.; Yao, J. L.; Wang, Y.; Zhou, K. R. Detection of small hepatocellular carcinoma: comparison of dynamic enhancement magnetic resonance imaging and multiphase multirow-detector helical CT scanning. World J. Gastroenterol., 2007, 13(8), 1252-6.

[48] Kim, S. R.; Ando, K.; Mita, K.; Fuki, S.; Ikawa, H.; Kanbara, Y.; Imoto, S.; Matsuoka, T.; Hayashi, Y.; Kudo, M. Superiority of CT arterioportal angiography to contrast-enhanced CT and MRI in the diagnosis of hepatocellular carcinoma in nodules smaller than $2 \mathrm{~cm}$. Oncology, 2007, 72 Suppl 1, 58-66.

[49] Gaiani, S.; Celli, N.; Piscaglia, F.; Cecilioni, L.; Losinno, F.; Giangregorio, F.; Mancini, M.; Pini, P.; Fornari, F.; Bolondi, L. Usefulness of contrast-enhanced perfusional sonography in the assessment of hepatocellular carcinoma hypervascular at spiral computed tomography. J. Hepatol., 2004, 41(3), 421-6.

[50] El-Serag, H. B.; Marrero, J. A.; Rudolph, L.; Reddy, K. R. Diagnosis and treatment of hepatocellular carcinoma. Gastroenterology, 2008, 134(6), 1752-63.

[51] Khan, M. A.; Combs, C. S.; Brunt, E. M.; Lowe, V. J.; Wolverson, M. K.; Solomon, H.; Collins, B. T.; Di Bisceglie, A. M. Positron emission tomography scanning in the evaluation of hepatocellular carcinoma. J. Hepatol., 2000, 32(5), 792-7.

[52] Porta, C.; De Amici, M.; Quaglini, S.; Paglino, C.; Tagliani, F.; Boncimino, A.; Moratti, R.; Corazza, G. R. Circulating interleukin6 as a tumor marker for hepatocellular carcinoma. Ann. Oncol., 2008, 19(2), 353-8. 